\title{
1242 - Application of Large-scale Synchrotron X-Ray Fluorescence 2D Mapping of Alteration Styles to Understand Gold Mineralization at the Monument Bay Project, Stull Lake Greenstone Belt, Manitoba, Canada
}

\author{
Juliana Casali, Chunyi Hao, Zohreh Ghorbani, Hannah Cavallin, Lisa Van Loon and Neil Banerjee
}

Western University, London, Ontario, Canada

The Monument Bay project is an Archean Greenstone-hosted gold deposit located $\sim 570 \mathrm{~km} \mathrm{NW}$ of Winnipeg, MB. The project is located within the Stull Lake Greenstone Belt, situated in the NW part of the Superior Province. Significant gold grades revealed in previous studies suggest that there is strong potential for the exploitation of gold reserves at the deposit [1]. The project area comprises three main rock groups (metavolcanic, felsic intrusive, and metasedimentary rocks) and six different prospecting target zones: Twin Lakes, Twin Lakes West, AZ, South Limb Shear, Mideast, and the Fence. Each target encompasses at least three different styles of hydrothermal alteration including carbonate, sericite, and chlorite alteration. The objective of this project is to delineate the mineralogical and geochemical footprint of the deposit by identifying and characterizing these alteration styles and their relationship to gold mineralization. It is important to understand the relationships between the targets to effectively vector towards future discoveries in underexplored regions of the property. Additionally, in developing a comprehensive understanding of the broad alteration footprint, the subsequent detection of gold mineralized zones can be applied on a district-wide scale. Chemical and mineralogical characteristics of the alteration footprint may help determine the proximity to high-grade $\mathrm{Au}$ mineralized zones. A combination of optical petrography, synchrotron radiation X-ray fluorescence (SR-XRF), and synchrotron radiation X-ray diffraction (SR-XRD) is being used to characterize in detail the chemical and mineralogical alteration footprint of the Monument Bay deposit.

Samples from half drill core were selected from all the prospecting targets to provide a representative suite of samples that covered the spatial distribution of the hydrothermal alteration. In addition, the samples were selected for different hydrothermal alteration characteristics, the presence of sulphides, and veining complexity. Samples showing diverse alteration styles and high gold grades were chosen for petrographic analyses. Optical petrography was conducted using a Nikon LV100POL microscope equipped with a Nikon DS-Ri1 digital camera at Western University. Petrography was used to identify the textures and mineralogical composition of the alteration styles and the major minerals. SR-XRD data collection on corresponding powders was conducted at the CMCF beamline 08BM-1 at the Canadian Light Source using established setup parameters for geologic materials [2]. SR-XRD was used to identify primary and alteration mineralogy.

SR-XRF was performed directly on the half drill core samples without extensive sample preparation at the 8-BM and 20-ID beamlines at Argonne National Laboratory (APS) in Illinois to create 2D trace element maps that reveal spatial and structural associations with gold mineralization. 2D SR-XRF maps of $10 \mathrm{~cm} \mathrm{x} 4 \mathrm{~cm}$ were produced by rastering across the sample, using a beam spot size of $800 \mu \mathrm{m} \times 800$ $\mu \mathrm{m}$ or $500 \mu \mathrm{m}$ x $500 \mu \mathrm{m}$ with a 2.5 second dwell time. The incident X-ray energy was set to 15 or $27 \mathrm{keV}$. A Vortex 4-element SDD was used to measure the XRF signal. SR-XRF data analysis was done with Peakaboo 5.3.0 [3]. Maps of key elements, such as Fe, As, and Au were compared to investigate geochemical associations, as well as macro-scale textures and alteration patterns, and associations of Au with other elements. 
Petrographic and SR-XRD results reveal that three main alteration styles are found at all six targets. Sericite and carbonate alteration (Figure 1) occur together in most of the rock types. Carbonate alteration is more predominant in the felsic intrusive rocks and metasedimentary rocks while sericite alteration is more common in metasedimentary rocks. Chlorite alteration is only evident in rocks that display higher amounts of deformation in the deposit. SR-XRF 2D element map results indicate Au is associated with carbonate and sericite alteration in all rock types and is consistently associated with the presence of arsenopyrite (e.g., Figure 2). K maps show that sericite alteration is present across the entire deposit, even in lithologies with very high carbonate alteration (values $>80 \%$ ).

SR-XRF element maps were used to interpret the structure of the samples as well as the spatial distribution between $\mathrm{Au}$ and other elements, using the elements as proxies for minerals and alteration. Sericite alteration is associated with the distribution of $\mathrm{K}$ maps. Correlated Fe and As in the element maps indicates the presence of arsenopyrite. Regions in the iron map not associated with other major elements are likely associated with pyrite mineralization. $\mathrm{Ca}, \mathrm{Mg}, \mathrm{Mn}$ and $\mathrm{Fe}$ commonly indicates the presence of carbonate minerals, $\mathrm{Pb}$ for galena, and $\mathrm{Zn}$ for sphalerite.

In conclusion, based on petrographic analysis, SR-XRD, and SR-XRF results an alteration pattern is observed that all the mineralized rocks are affected by carbonate and sericite alteration. These results indicate that all the prospecting targets are located inside the mineralized footprint of the deposit. However, the different relationships between $\mathrm{Au}$ and the sulphide minerals are likely an indication of several different mineralization events.

Acknowledgements: Research described in this paper was performed at the Canadian Light Source (CLS) and Advanced Photon Source (APS). The CLS is supported by the CFI, NSERC, the Univ. of Saskatchewan, the Government of Saskatchewan, Western Economic Diversification Canada, the NRC, and the CIHR. The APS is an Office of Science User Facility operated for the U.S. Department of Energy (DOE) Office of Science by Argonne National Laboratory, and is supported by the U.S. DOE under Contract No. DE-AC02-06CH11357, and the CLS and its funding partners. The authors acknowledge funding from NSERC and Yamana Gold Inc. We thank M. Fodje, and S. Labiuk, Z. Finfrock, D. M. Meira, and E. Maxey for support in the experiments.
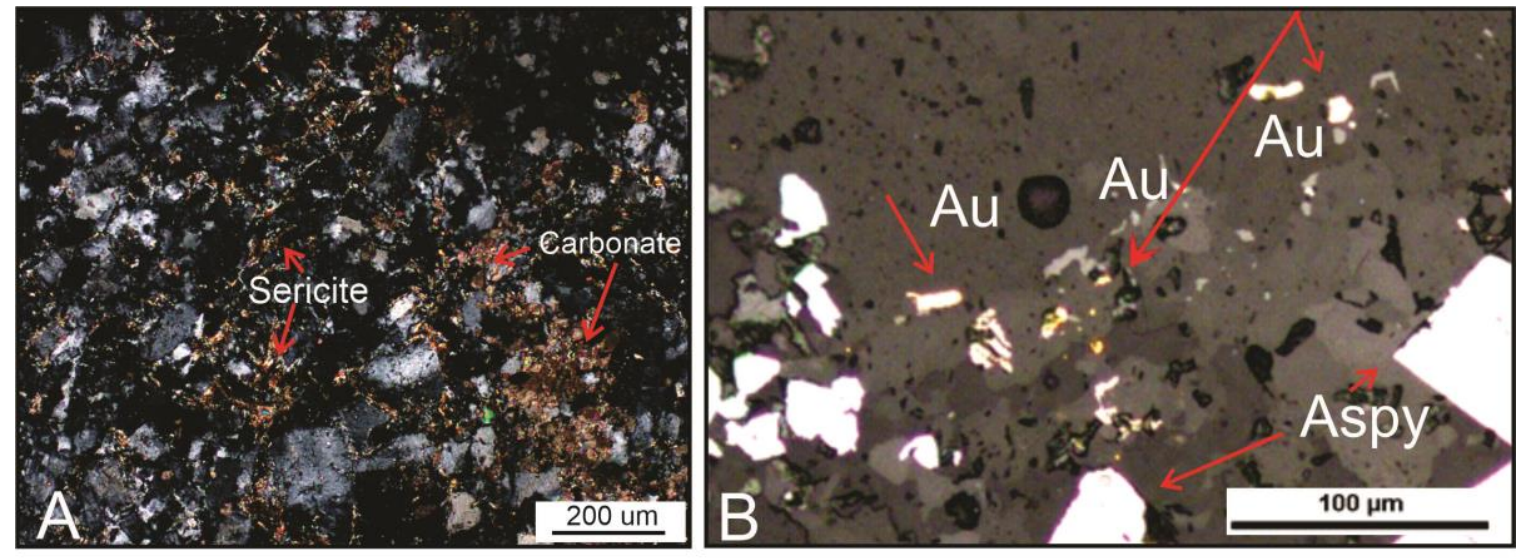
Figure 1. Photomicrographs showing (A) the quartz-feldspar matrix with sericite and carbonate alteration. (B) the distribution of $\mathrm{Au}$ and arsenopyrite (Aspy). The red arrows point to the minerals described displayed in the photomicrographs.

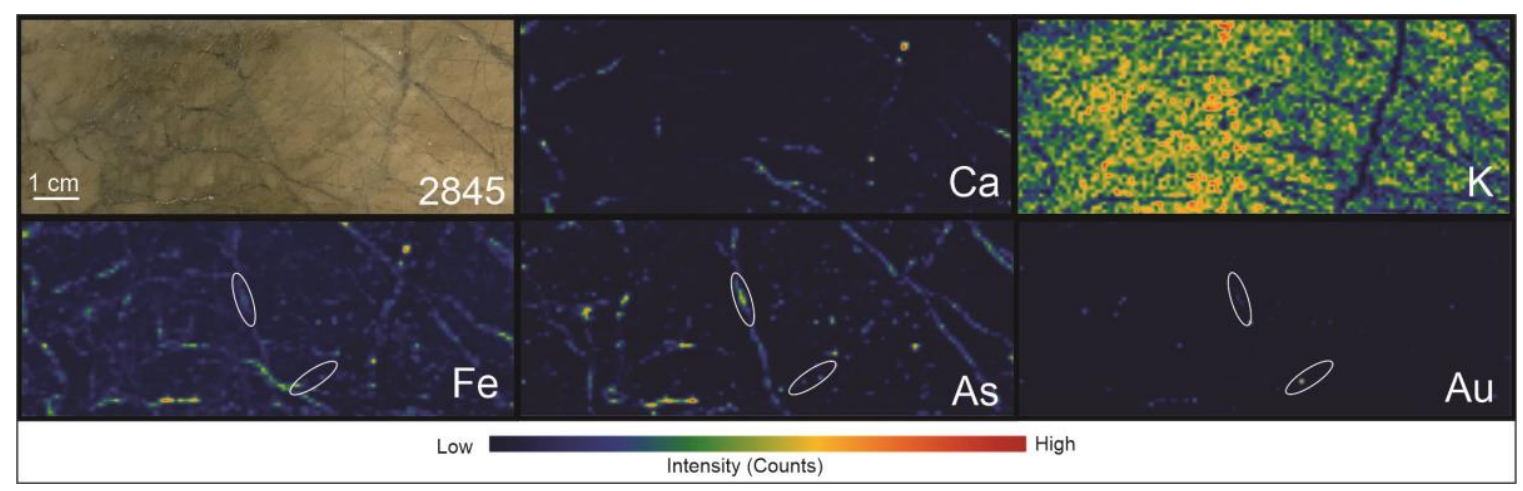

Figure 2. Examples of SR- $\mu$ XRF element maps collected for a single sample from the Twin Lakes West zone showing major elements $\mathrm{Fe}$ and As associated with gold mineralization. The $\mathrm{K}$ map represents sericite alteration. The Ca map represents both carbonate alteration and carbonate veins. White circles show the spatial relationship between Fe and As (interpreted as arsenopyrite), and Au located with Fe and As.

\section{References}

[1] T. McCraken et al., "Technical Report and Resource Estimate Update on the Monument Bay Project", Unpublished.

[2] N. Banerjee et al, Microscopy \& Microanalysis 24 (Suppl2) (2018) p.518.

[3] L.L Van Loon et al, Peakaboo: Advanced software for the interpretation of X-ray fluorescence spectra from synchrotrons and other intense X-ray sources. Software Impacts, 2 (2019). 